\title{
Protocol
}

\section{The South African macular oedema in diabetics study (the same study): a prospective randomized non-inferiority trial}

\author{
Naseer Ally ${ }^{1,2 *}$, Sarah Ismail ${ }^{1,2}$, Bongi P. Sithole ${ }^{1,2}$, Mpho Tsimanyane ${ }^{1,2}$, Ismail Mayet ${ }^{1,2}$, \\ Tebogo R. Kgarebe ${ }^{3}$, Angelika U. Carey ${ }^{1,2}$, Taimeia G. Hussain ${ }^{1,2}$, Craig D. Anderson 1,2, \\ Kihagi Mwita1 $^{1}$, Kashmira Rawjee ${ }^{1,2}$, Monique D. G. Camacho, ${ }^{1,2}$, Melody W. Sun ${ }^{1,2}$, \\ Thabiso Mofokeng ${ }^{1,2}$, Fathima Mitha ${ }^{1,2}$, Lintle Ntlou ${ }^{1}$, Ingrid Walters ${ }^{1,2}$, Hester Kruger ${ }^{1,2}$, \\ Farai N. F. Mabunda ${ }^{1}$, Lieschen Branders ${ }^{1}$, Ismail Makda ${ }^{1,2}$, \\ Wihan de Jager ${ }^{1,2}$, Hassan D. Alli1 ${ }^{2}$
}

\begin{abstract}
${ }^{1}$ St John Eye Hospital, Chris Hani Road Diepkloof, Soweto
${ }^{2}$ University of the Witwatersrand, Department of Neurosciences, Division of Ophthalmology, York road Parktown Johannesburg

${ }^{3}$ St John Eyecare Clinic, Chris Hani Road, Soweto
\end{abstract}

Received: 16 September 2020

Accepted: 08 October 2020

\author{
*Correspondence: \\ Dr. Naseer Ally, \\ E-mail: Naseer.Ally@wits.ac.za
}

Copyright: (c) the author(s), publisher and licensee Medip Academy. This is an open-access article distributed under the terms of the Creative Commons Attribution Non-Commercial License, which permits unrestricted non-commercial use, distribution, and reproduction in any medium, provided the original work is properly cited.

\begin{abstract}
Background: Diabetic retinopathy is the most common cause of visual loss affecting the economically productive age group globally. Diabetic macular oedema (DMO) results from leakage of fluid into the retinal interstitial space. Antivascular endothelial growth factor (anti-VEGF) drugs are the first line treatment for DMO. Since monthly injections are required, this treatment regimen can prove very costly. Of the anti-VEGF drugs, bevacizumab is the most costeffective. The pro re nata (PRN) method is the current standard of care. However, the treat and extend (T and E) regimen can potentially decrease the patient burden on hospitals. Thus far, no randomised clinical trials have been performed using Bevacizumab in a treat and extend versus pro re nata regimen.

Methods: A prospective randomised non-inferiority clinical trial testing bevacizumab (1.25 $\mathrm{mg})$ in a treat and extend method versus the pro re nata method is being conducted. Patients will be randomised using a simple computer-based randomised algorithm. The primary outcome is non-inferiority within a five-letter margin for the $\mathrm{T}$ and $\mathrm{E}$ regimen versus the PRN regimen.

Conclusions: This study aims to inform a key area in the literature on the treatment of DMO, i.e. whether a $\mathrm{T}$ and $\mathrm{E}$ regimen is non-inferior to a PRN regimen in the treatment of DMO with bevacizumab, which is the only anti VEGF available in resource poor settings. It is motivated by the cost involved in the treatment of DMO as well as the treatment burden on both the patient and the health institution at which the patient is receiving treatment.

Trial registration: The trial has been registered on the Pan-African clinical trials registry (PACTR202001624880753).
\end{abstract}

Keywords: Bevacizumab, Diabetic macular oedema, Pro re nata, Treat and extend, Diabetic retinopathy, Anti-VEGF

\section{INTRODUCTION}

The prevalence of diabetes mellitus (DM) has increased worldwide, more than doubling in the past 3 decades. ${ }^{1}$ In
Africa, it is projected to increase by $98 \%$ from 12,1 million in 2010 to 23,9 million in 2030 . This is due to factors such as urbanisation, an aging population and lifestyle changes. $^{1}$ Diabetic retinopathy (DR), one of the 
complications of DM, is the most common cause of visual loss among income-generating adults around the world. ${ }^{2}$ Diabetic macular oedema (DMO) is a leading cause of this visual loss among the diabetic population. ${ }^{3}$ DMO results from a disruption of the blood-retina barrier and leakage of fluid into the interstitial space. ${ }^{3}$ The processes resulting in this leakage are multifactorial, and may involve an increase in vascular permeability, activation of inflammatory cytokines, alterations in the dynamics of retinal vascular flow and hypoxia.

Numerous treatment modalities have been used for the treatment of diabetic macular oedema, which include laser photocoagulation, intravitreal steroid injections and intravitreal anti-vascular endothelial growth factor (antiVEGF) drugs. ${ }^{4-7}$ Anti-VEGF drugs have emerged as the first-line treatment for patients with DMO. ${ }^{8}$ There are currently 3 anti-VEGF drugs that are in general use, namely Ranibizumab (Lucentis $\AA$, Genentech/Roche), Aflibercept (Eyelea ${ }^{\circledR}$, Regeneron/Bayer HealthCare), and off-label Bevacizumab (Avastin ${ }^{\circledR}$, Genentech/Roche) ${ }^{6}$ These injections are usually given into the eye on a monthly basis, necessitating repeated hospital visits. Even though anti-VEGF treatment is first-line, it is associated with a much higher cost when compared to other treatment modalities. ${ }^{9}$ This problem is more pronounced in Africa and other developing countries where resources for eye care are severely constrained.

In developing countries, especially in sub-Saharan Africa there is a shortage of trained ophthalmologists. ${ }^{10}$ This results in specialist eyecare services not being readily available everywhere, especially in rural areas. Many patients are therefore only able to access healthcare at a few centres to receive their treatment. Due to this burden on eyecare services, a new way to deliver the optimal outcomes for patients needs to be sought (i.e. ways that maximise visual outcomes whilst lowering patient cost and hospital service costs). The treat and extend (T\&E) regimen of anti-VEGF use has been shown to be effective in other conditions that result in leakage of fluid at the macula such a neovascular age-related macular degeneration. ${ }^{11}$ In this treatment regimen, patients are brought less frequently to the hospital and the time interval between treatments increases once their central foveal subfield thickness decreases. This is a favourable method to address the aforementioned cost and healthcare burden issues. The $\mathrm{T}$ and $\mathrm{E}$ regimen has been tested against the PRN regimen in DMO using Ranibizumab with proven non-inferior outcomes. ${ }^{12}$

Due to cost constraints, however, Bevacizumab is the treatment of choice in Africa and many other developing countries. To the authors' knowledge there has thus far been no study comparing Bevacizumab using the PRN regimen to the $\mathrm{T}$ and $\mathrm{E}$ regimen. There has also been no prospective clinical trial using anti-VEGF to treat DMO in a cohort of patients in sub-Saharan Africa. This will be especially pertinent to developing countries in Africa and elsewhere.

\section{METHODS}

\section{Study design}

The South African macular edema (SAME) study is a prospective randomized non-inferiority clinical trial. The aim of this study is to compare the efficacy and costeffectiveness of two treatment regimens ( $T$ and $E$ versus PRN) using intravitreal Bevacizumab in patients with DMO at St John Eye Hospital (SJEH). The trial is being conducted in accordance with the tenets of the declaration of Helsinki.

After obtaining informed consent, patients will undergo the following examinations: uncorrected distance visual acuity (UDVA) and corrected distance visual acuity (CDVA) measured in logMAR and slit-lamp biomicroscopy examination. They will also have the following investigations: fluorescein angiography (FA), macular optical coherence tomography (OCT) using the Spectralis (Heidelberg Engineering, Germany) spectral domain optical coherence tomography (SD-OCT) and the following blood tests: glycated haemoglobin (HbA1C), urea and electrolytes (U and E), lipogram and full blood count (FBC) at baseline. Only one eye per patient will be enrolled in the study. The eye to be included in the study is the eye with the poorer baseline visual acuity (VA). This is for safety reasons and in order for the eye with the worse baseline visual acuity to receive the first injection at the visit where all baseline investigations are conducted. Patients will then undergo randomisation using a simple, computer-based randomisation algorithm into either $\mathrm{T}$ and E or PRN dosing cohorts. Both treatment groups (T and E and PRN) will initially receive 3 doses of Bevacizumab (1.25 mg at each visit) at monthly intervals i.e. one dose per month for 3 months. At each of these and subsequent visits they will undergo UDVA and CDVA testing, slitlamp biomicroscopic examination, and OCT. After the 3 initial doses, they will continue with the T\&E or PRN treatment (figure1).

Patients in the T\&E treatment arm will be assessed at the 4-month visit. Patients who have no macular oedema or other signs of exudation on OCT at 4 months, are injected at the 4-month visit, and instructed to return in 6 weeks. If they have macular oedema or other signs of exudation, they are given an injection at the 4-month visit and told to return in 4 weeks for assessment. Patients returning after 6 weeks will be assessed again for macular oedema on OCT. If there are no signs of macular oedema on OCT, they are given an injection and told to return in 8 weeks. If they have macular oedema on OCT, they are given an injection and told to return in 4 weeks. This cycle repeats itself until the optimal duration of treatment per patient is achieved (a patient has a decrease in duration at least twice after increases and is stable at the new duration) to a maximum interval between follow ups of three months (Figure 1).

After receiving the three-monthly injections, those patients in the PRN group will follow up monthly and receive more 
avastin injections if there is evidence of fluid on macular OCT. If there is no fluid on OCT they will not be injected and come for a follow-up visit in one month. If they are injected, they will also follow up in one month. The PRN group will follow up monthly for the remainder of the study whether they have macular oedema or not (Figure 1).

Rescue grid laser may be performed from month 6 onwards at the discretion of the blinded investigators (IM and HDA), if there is no response to intravitreal bevacizumab injections.

Blood tests including the FBC, $\mathrm{U}$ and E, Lipogram and $\mathrm{HbA1C}$ will be collected 6-monthly for the duration of the study. Economic factors such as duration of time spent traveling to and from the hospital, money spent on transportation and kilometres travelled will also be collected at each visit.

The primary outcome will be measured at 1 year. The study investigators have been separated into exclusive groups to maintain blinding during the measurements of the visual acuity and OCTs. All refractions will be carried out by a single optometrist (RM) who is blinded to the study groups. Group 1 (SI, BPS, MT, AC, TGH, CDA, KM, KR, LN, HK, FM, LB, IMak) will perform the OCTs, FAs and intravitreal injections. All decisions regarding treatment, i.e. to inject or not in the PRN group and to extend or not in the $\mathrm{T}$ and $\mathrm{E}$ group, will be made by group 2 (IM and HDA) who are blinded to the study groups. They will only be shown the OCTs (by group 3 ) to determine if macular oedema is present or not. Group 3 (NA, MWS, MC, FM, TM, WdJ) who are unblinded, are involved in the assessments of the patients and carrying out the decisions made by HDA and IM. To summarise, all investigations and injections will be performed by group 1 who are blinded to the patient groups. All treatment decisions are made by group 2 who are also blinded. Group 3 is unblinded and are responsible for clinical assessment. No outcome measures besides cost of transport are directly collected by group 3 .

\section{Intervention}

Bevacizumab (Avastin $®$, Genentech/Roche) is a monoclonal antibody that inhibits all isoforms of VEGFA. It is used off-label and is the first line treatment for the treatment of macular oedema at all public hospitals in South Africa. At St John Eye Hospital it is the only antiVEGF drug available for the treatment of macular oedema.

\section{Primary objective}

The primary objective of the SAME study is to determine if the T\&E regimen is non-inferior to the PRN regimen when comparing visual outcomes (the number of letters changed between baseline and the 1-year visit) with a fiveletter margin of non-inferiority at 1 year. The visual outcomes will be assessed by documenting the number of letters changed from baseline to the one-year visit, or the closest visit to one year in the case of the T\&E arm. The visual acuity will be measured using a standard Early treatment of diabetic retinopathy study (ETDRS) visual acuity chart. The UDVA and CDVA will be recorded in $\log$ MAR.

\section{Secondary objectives}

The secondary outcomes of the SAME study are: comparing the $\mathrm{T}$ and $\mathrm{E}$ regimen to the $\mathrm{PRN}$ regimen in terms of the anatomical changes in the central foveal subfield thickness and to determine the economic impact on patients of the two regimens by looking at the financial impact of the hospital visits on the patients. The former will be modelled by taking the change in the mean central subfield thickness which is measured by using the standard ETDRS grid on the Spectralis SD-OCT (Heidelberg Engineering, Germany) and comparing the changes between the two groups. This will be done for noninferiority. The economic impact will entail a direct comparison between the two groups of the mean amount of money and time spent travelling to and from the hospital over the course of the study.

\section{Setting}

The South African Macular oedema in diabetics Study (The SAME Study) is being conducted at St John Eye Hospital (SJEH) located in Soweto, Johannesburg, South Africa. St John Eye Hospital is the Ophthalmology Department of Chris Hani Baragwanath Academic Hospital (CHBAH). The largest eye hospital on the African continent, and is located in the province of Gauteng which is the smallest, yet most densely populated province. It primarily serves patients from the nearby township of Soweto with an estimated population of over 1,2 million people. ${ }^{13}$ Since it is one of the few tertiary hospitals in the country, the hospital also drains patients from the surrounding rural provinces as well as neighbouring countries of Zimbabwe, Mozambique, Lesotho and Swaziland.

\section{Inclusion criteria}

All diabetic patients with confirmed DMO- associated visual impairment and the following: Snellen corrected distance visual acuity (CDVA) of 6/9 or worse; and CFT $\geq 250 \mu \mathrm{m}$ on the Spectralis SD-OCT (Heidelberg engineering) OCT (quality of OCT must be $>20$ ).

\section{Exclusion criteria}

The exclusion criteria for the study is as follows: patients who have previously received treatment for DMO (antiVEGF; grid laser; or periocular or intravitreal steroids), macular oedema from any other causes such as vein occlusion, post cataract surgery and uveitis, patients with epiretinal membranes identified either on clinical examination or on OCT, patients with media opacities precluding an adequate view of their fundus or resulting in 
poor quality OCT scans, patients with macular ischaemia on fluorescein angiography and patients with other causes of poor vision such as corneal opacities, cataracts, media opacities, optic nerve dysfunction, retinal detachments and other macular/retinal pathologies.

\section{Time frame}

The study commenced in November 2017. The expected duration of enrolment is 3 years with a one year follow up for the primary outcome. The study is expected to complete data collection for the primary outcome by December 2021.

\section{Sample size}

The sample size was calculated using a one-sided two sample t-test with a null hypothesis of the $T \& E$ regimen being inferior to the PRN regimen:

$\mathrm{H} 0: \mu T \& E-\mu P R N>5$ letters

The alternative hypothesis is that the T\&E regimen is noninferior to the PRN regimen.

Ha: $\mu T \& E-\mu P R N \leq 5$ letters

Using the above test with a non-inferiority margin of 5 letters (1 line on a LogMAR VA chart), a standard deviation of 10 letters, an alpha-level of 0.05 , and a power of $80 \%$, we calculated a sample size of 102 patients. We added an anticipated dropout rate of $15 \%$ and ended up with a total sample size of 117 which we then rounded up to 120 patients.

\section{Data collection}

The following data will be collected at baseline $(*)$ and follow-up visits (+): patient age, sex, duration of diabetes, other medical co-morbidities, place of residence*, UDVA and $\mathrm{BCVA}^{*}+$, CFT on OCT and OCT quality*+, FA findings*, $\mathrm{FBC}$, lipogram, $\mathrm{HbA} 1 \mathrm{c}$ and $\mathrm{U}$ and $\mathrm{E}^{*}(\mathrm{At}$ baseline and 6-monthly thereafter), amount of money spent on trip to hospital*+, duration of trip to the hospital*+ and whether a patient is accompanied to the hospital by a family member*+.

All data will be entered onto the Research Electronic Data Capture (REDCap) hosted at the University of the Witwatersrand. ${ }^{14,15}$ The data will be entered by NA and FM.

\section{Statistical analysis}

Statistical analysis will be carried out using Stata 16.1 (STATACorp, College Station, Texas). Statistical significance will be set at 0.05 . Descriptive statistics such as the mean and standard deviation will be used for continuous variables, and frequency distribution and percentages will be used for categorical variables at baseline.

Missing data will be accounted for using multiple imputation with chained equations since we expect the pattern of missingness to be non-monotone. Missing baseline characteristics will be imputed using mean imputation. We will then use a mixed effects linear regression model to model the primary outcome. If the lower end of the $95 \%$ confidence interval (CI) of the $\mathrm{T}$ and E group is within the 5-letter non-inferiority margin, then the $\mathrm{T}$ and $\mathrm{E}$ group will be deemed to be non-inferior to the PRN group.

\section{Ethical considerations}

Ethical clearance has been obtained from the University of the Witwatersrand Human Research Ethics Committee. Informed consent will be obtained from patients with translators if required. Oversight of adverse reactions will be performed by the University of the Witwatersrand human research ethics committee.

\section{Confidentiality}

Each patient's hospital number will be assigned a unique code. This code will appear on the data collection sheet together with the information obtained from the patients file. The file linking the patient code to the patient hospital number will be kept separate from the datasheet and will only be used for crosschecking purposes.

\section{Trial registration}

The trial has been registered on The Pan-African Clinical Trials Registry (PACTR202001624880753).

\section{Potential risks to patients}

There is a small risk of complications associated with intravitreal Bevacizumab injection. These include endophthalmitis (severe eye infection), cataract, vitreous haemorrhage and retinal detachment. ${ }^{16}$ These will be managed accordingly by the doctors at St John Eye Hospital. To minimize this risk, the injection will be carried out using a sterile technique under sterile conditions as per standard protocols. The fluorescein dye used in the fluorescein angiogram (FA) test usually causes a yellow discolouration of the skin that lasts 6-12 hours and orange-yellow discolouration of the urine that can last 24-36 hours. More severe allergic reactions such as nausea or vomiting can also occur in $0.2-8 \% .{ }^{17}$ Skin rashes (urticaria) occur in less than $0.2-4.8 \%$ of patients. Anaphylactic reactions occur in $0.03-0.38 \%$ of patients. If there is extravasation of the dye it can cause skin inflammation and necrosis, although this is extremely rare. If an allergic or anaphylactic reaction does occur, it will be managed by the doctors at St John Eye Hospital, with help from internists as dictated by the severity. 


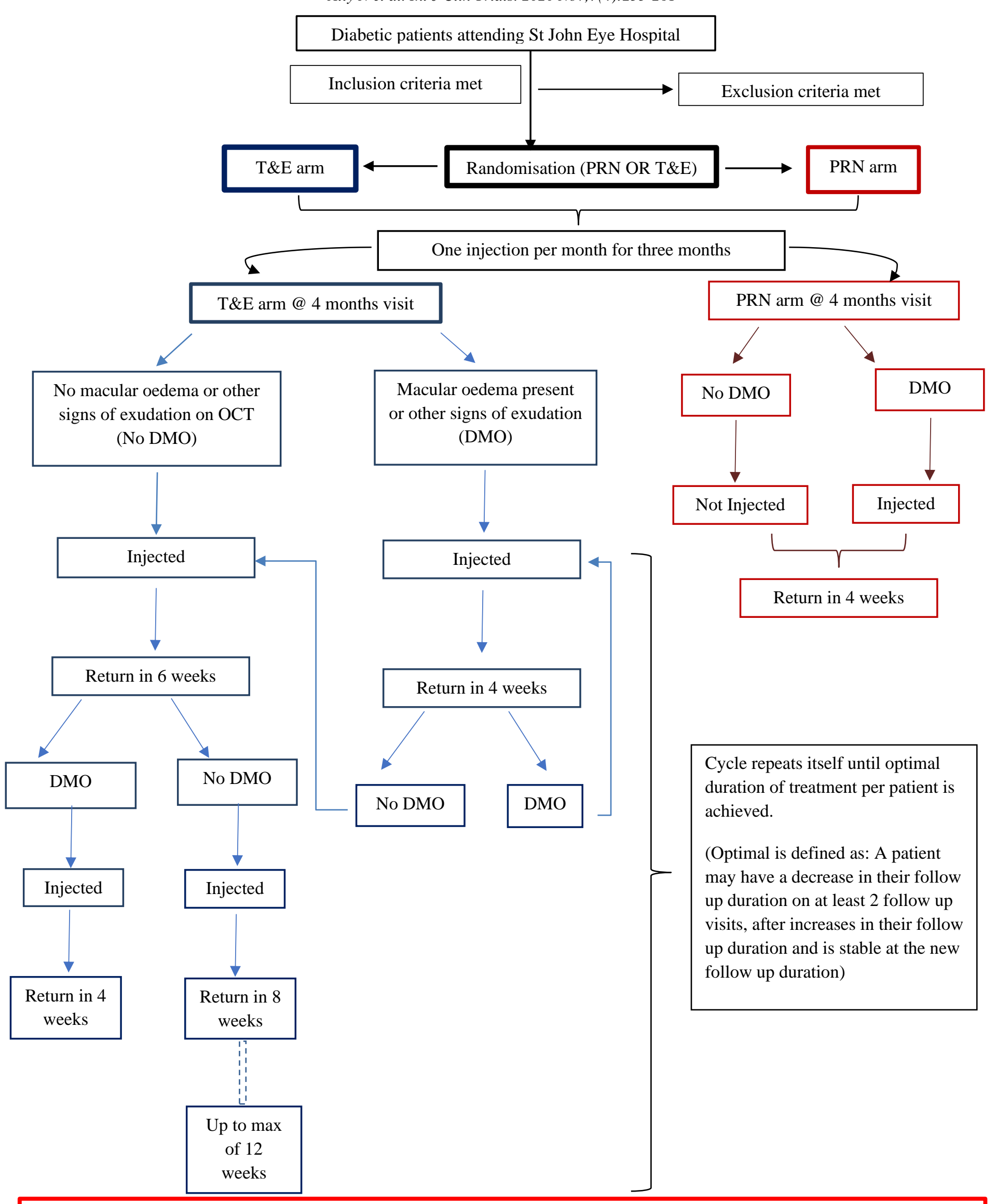

Rescue laser can be performed in both arms from month 6 onwards blood tests every 6 months

Figure 1: Patient recruitment, enrolment, and study procedures. 


\section{DISCUSSION}

This study aims to fill in a key area in the literature on the treatment of DMO, i.e. whether a T\&E regimen is noninferior to a PRN regimen in the treatment of DMO with Bevacizumab. This is often the only anti-VEGF available in resource poor settings. It is motivated by the cost involved in the treatment of DMO as well as the treatment burden on both the patient and the health institution at which the patient is receiving treatment. Bevacizumab has been shown to be the most cost-effective of all the antiVEGF treatments. ${ }^{18}$ This coupled with a T and E regimen provides a good value proposition in the treatment of DMO in resource constrained settings. Although this study does not aim to look at this, there are also other benefits to the $\mathrm{T}$ and $\mathrm{E}$ regimen when compared to the $\mathrm{PRN}$ regimen. This includes less psychological stress as patients in the $\mathrm{T}$ and E regimen know that they will receive an injection at every visit. ${ }^{19}$ In contrast, the patients treated according to the PRN regimen only know whether they will be injected once they have had an examination, OCT and visual acuity test.

There are some limitations to this study. Firstly, it is a single centre study and that may introduce some bias. This also results in a slower enrolment rate. A single centre, however, may result in better trial oversight. Secondly, the trial data is not being sent to a reading centre and this may introduce some bias as well. To this end, we have kept the groups in the study separate to maintain blinding. The patients are, however, not blinded and this cannot be maintained. Since the patients are from lower socioeconomic areas, and they pay for their own transport we felt that sham visits would be unethical.

Finally, it is also important to note that this study is the first study of its kind to be conducted on the African continent. Most data thus far have been produced by developed countries and we believe that appropriate management solutions need to be investigated in and adopted by developing countries.

\section{CONCLUSION}

The SAME study is the first non-inferiority trial using Bevacizumab in the treatment of DMO. This is especially important for resource poor settings and developing countries.

\section{ACKNOWLEDGEMENTS}

The authors wish to thank Dr T. Jeeva-Patel and Dr T. Bagus for their assistance with the study.

Funding: This study is being funded in part by the University of the Witwatersrand Faculty research grant Conflict of interest: None declared

Ethical approval: The study was approved by the Institutional Ethics Committee

\section{REFERENCES}

1. Chen L, Magliano DJ, Zimmet PZ. The worldwide epidemiology of type 2 diabetes mellitus- present and future perspectives. Nat Rev Endocrinol. 2012;8(4):228-36.

2. Klein BEK. Overview of epidemiologic studies of diabetic retinopathy. Ophthalmic Epidemiol. 2007;14(4):179-83.

3. Ford JA, Lois N, Royle P, Clar C, Shyangdan D, Waugh N. Current treatments in diabetic macular oedema: systematic review and meta-analysis. BMJ Open. 2013;3(3).

4. Photocoagulation for diabetic macular edema. Early Treatment Diabetic Retinopathy Study report number 1. Early Treatment Diabetic Retinopathy Study research group. Arch Ophthalmol Chic Ill 1960. 1985;103(12):1796-806.

5. Nguyen QD, Shah SM, Khwaja AA, Channa R, Hatef E, Do DV, et al. Two-year outcomes of the ranibizumab for edema of the mAcula in diabetes (READ-2) study. Ophthalmol. 2010;117(11):214651 .

6. Wells JA, Glassman AR, Ayala AR, Jampol LM, Bressler NM, Bressler SB, et al. Aflibercept, Bevacizumab, or Ranibizumab for Diabetic Macular Edema: Two-Year Results from a Comparative Effectiveness Randomized Clinical Trial. Ophthalmol. 2016;123(6):1351-9.

7. Grover DA, Li T, Chong CC. Intravitreal steroids for macular edema in diabetes. Cochrane Database Syst Rev. 2008. Available at: http://www.cochranelibrary.com/cdsr/doi/10.1002/14651858.CD005656. pub2/full. Accessed on 17 August 2020.

8. Anti-vascular endothelial growth factor for diabetic macular oedema: a network meta-analysis - Virgili, G 2018. Cochrane Library. 2018. Available at: https://www.cochranelibrary.com/cdsr/doi/10.1002/ 14651858.CD007419.pub6/full. Accessed on 17 August, 2020.

9. Romero-Aroca P, Riva-Fernandez S de la, VallsMateu A, Sagarra-Alamo R, Moreno-Ribas A, Soler $\mathrm{N}$, et al. Cost of diabetic retinopathy and macular oedema in a population, an eight year follow up. BMC Ophthalmol. 2016;16(1):1-7.

10. Resnikoff S, Lansingh VC, Washburn L, Felch W, Gauthier T-M, Taylor HR, et al. Estimated number of ophthalmologists worldwide (International Council of Ophthalmology update): will we meet the needs? Br J Ophthalmol. 2020 ;104(4):588-92.

11. Chin-Yee D, Eck T, Fowler S, Hardi A, Apte RS. A systematic review of as needed versus treat and extend ranibizumab or bevacizumab treatment regimens for neovascular age-related macular degeneration. Br J Ophthalmol. 2015;100(7):914-7.

12. Prünte C, Fajnkuchen F, Mahmood S, Ricci F, Hatz $\mathrm{K}$, Studnička J, et al. Ranibizumab $0.5 \mathrm{mg}$ treat-andextend regimen for diabetic macular oedema: the RETAIN study. Br J Ophthalmol. 2016;100(6):78795. 
13. Main Place. Statistics South Africa. Available at: http://www.statssa.gov.za/?page_id=4286\&id=1131 7. Accessed on 17 August, 2020.

14. Harris PA, Taylor R, Thielke R, Payne J, Gonzalez $\mathrm{N}$, Conde JG. Research electronic data capture (REDCap) - A metadata-driven methodology and workflow process for providing translational research informatics support. J Biomed Inform. 2009;42(2):377-81.

15. Harris PA, Taylor R, Minor BL, Elliott V, Fernandez M, O'Neal L, et al. The REDCap consortium: Building an international community of software platform partners. J Biomed Inform. 2019;95:103208.

16. Falavarjani KG, Nguyen QD. Adverse events and complications associated with intravitreal injection of anti-VEGF agents: a review of literature. Eye Lond Engl. 2013;27(7):78794.
17. Kornblau IS, El-Annan JF. Adverse reactions to fluorescein angiography: A comprehensive review of the literature. Surv Ophthalmol. 2019;64(5):679-93.

18. Maniadakis N, Konstantakopoulou E. Cost Effectiveness of Treatments for Diabetic Retinopathy: A Systematic Literature Review. PharmacoEconomics. 2019;37(8):995-1010.

19. Senra H, Ali Z, Balaskas K, Aslam T. Psychological impact of anti-VEGF treatments for wet macular degeneration-a review. Graefes Arch Clin Exp Ophthalmol Albrecht Von Graefes Arch Klin Exp Ophthalmol. 2016;254(10):1873-80.

Cite this article as: Ally N, Ismail S, Sithole BP, Tsimanyane M, Kgarebe TR, Mayet I, Carey AU et al. The South African macular oedema in diabetics study: a prospective randomized non-inferiority trial. Int J Clin Trials 2020;7(4):255-61. 